\title{
MEJORAMIENTO DE LA COMPETENCIA COMUNICATIVA EN ESPAÑOL COMO LENGUA EXTRANJERA A TRAVÉS DE LA VIDEOCOMUNICACIÓN*
}

\section{IMPROVEMENT OF THE COMMUNICATIVE COMPETENCE IN SPANISH AS A FOREIGN LANGUAGE BY USING VIDEO COMMUNICATION}

ANA VINE JARA

Universidad de Concepción. Concepción, Chile

avine@udec.cl

\section{ANITA FERREIRA CABRERA}

Universidad de Concepción. Concepción, Chile

aferreir@udec.cl

\section{RESUMEN}

La competencia comunicativa, entendida como la capacidad de una persona para producir y comprender mensajes adecuados, tanto en el contexto de las temáticas tratadas como en las distintas situaciones comunicativas, ha sido poco estudiada experimentalmente por la dificultad que representa. En este estudio, el propósito principal es visualizar cómo las tareas cooperativas interculturales en el ámbito de la videocomunicación como recurso tecnológico, esto es, uso del chat, cámara web y micrófono, mejoran la competencia comunicativa de estudiantes de español como lengua extranjera (ELE) en una modalidad blended-learning. De tal manera que para probar su efectividad se ha diseñado un estudio experimental con pre y postest con grupo control. Los resultados muestran que el aprendizaje logrado por el grupo experimental que realizó las tareas en el contexto de la videocomunicación fue superior al aprendizaje del grupo control que desarrolló las tareas utilizando el chat.

Palabras clave: Videocomunicación, chat, tarea cooperativa intercultural, competencia comunicativa.

* El estudio empírico que se presenta en este artículo está desarrollado en el contexto del Proyecto de investigación FONDECYT 1110812 "Un SistemaTutorial Inteligente para la focalización en la forma en la enseñanza del Español como Lengua Extranjera". 


\begin{abstract}
The concept of communicative competence, understood as the skills a person develops to be able to understand and produce messages suitable to the different topics and communicative situations encountered, has not been thoroughly studied through experimental research, due to its difficulty. The purpose of this study is to provide evidence of the positive effects that intercultural cooperative video-web communication tasks (using chat, web cam and microphone) have in the development of communicative competence of students learning Spanish as a foreign language (SFL) in a b-learning course. Thus, an experimental study with a pretest - postest and control group was designed to demonstrate the effectiveness of this kind of pedagogical practices. The results show the experimental group, which carried out the tasks through video web interaction, achieved a higher language learning level than the control group which performed the tasks using the chat.
\end{abstract}

Keywords: Video-web communication, chat, intercultural cooperative tasks, communicative competence.

Recibido: 10.12.2011. Aceptado: 29.04.2012.

\title{
1. INTRODUCCIÓN
}

\begin{abstract}
A ctualmente, los avances tecnológicos en el ámbito de la Lingüística Aplicada Ay, específicamente, en la Adquisición y Enseñanza de Segundas Lenguas permiten llevar a cabo nuevos desarrollos metodológicos de enseñanza y adquisición de lenguas. Esto ha llevado a investigadores y profesores de esta área a explorar y probar empíricamente nuevas herramientas para determinar los aportes en el ámbito del aprendizaje de lenguas extranjeras y segundas lenguas. Lo anterior constituye un desafío para quienes deseen utilizar estos recursos con propósitos educativos y/o investigativos, ya que deben ser capaces de manejarlos para luego generar una propuesta que sea efectiva, de modo que pueda considerarse un aporte a la disciplina y una mejora en el proceso de enseñanza de una segunda lengua.

En los últimos años, el uso masivo de las TICs (Tecnologías de la Información y Comunicación) ha enriquecido el proceso de enseñanza-aprendizaje de lenguas. Esto se ve reflejado en la enseñanza del español como lengua extranjera, a través del uso de diferentes herramientas tecnológicas, tales como: chat, blog, foros, videoconferencia, etc., utilizadas en diferentes modalidades de enseñanza, ya sea a distancia o semipresencial (blended learning). En este contexto se sitúa la videocomunicación o comunicación oral sincrónica a través de la web. Este tema ha sido ampliamente estudiado por distintos investigadores (Jauregi, Canto y Ros, 2006; Jauregi y Bañados, 2008; Juan, 2009), primeramente en una fase piloto y luego en contextos experimentales.
\end{abstract}


La problemática en cuestión, mejoramiento de la competencia comunicativa, ha sido poco investigada en la enseñanza de lenguas mediatizada por la tecnología, debido a la dificultad con que se encuentran los docentes para evaluar el progreso logrado por el estudiante (Blake, Karp y Barela, 1999), principalmente, en una modalidad semipresencial. Sin embargo, con el avance tecnológico hoy existen herramientas que permiten mejorar las dificultades que presenta la enseñanza de lenguas en una modalidad presencial, que muchas veces se centra en aspectos gramaticales de la lengua y aun cuando se dan las oportunidades para que los estudiantes practiquen la lengua, esto ocurre en un tiempo limitado por la duración de la clase, número de alumnos del curso, programa de la asignatura, etc.

En este artículo, el objetivo principal es visualizar cómo las tareas cooperativas interculturales realizadas a través de videocomunicación mejoran la competencia comunicativa de los estudiantes de ELE de nivel intermedio en una modalidad blended learning. Para ello, se operacionalizó el enfoque basado en tareas en un ambiente mediatizado por la videocomunicación que ofrece Skype, un software que permite la comunicación virtual entre los hablantes.

\section{MARCO DE REFERENCIA}

A continuación se presentan los conceptos y fundamentos teóricos en que se circunscribe la problemática en estudio.

\subsection{CALL (Computer Assisted Language Learning)}

Esta investigación se enmarca en el ámbito de la Lingüística Aplicada, específicamente en CALL (del inglés, Computer Assisted Language Learning), una interdisciplina en rápida evolución que se dedica a explorar el rol del computador y de las tecnologías de la información y de la comunicación en el aprendizaje y enseñanza de lenguas (Ferreira, 2007). Utilizar una aplicación en el ámbito de CALL presenta una serie de ventajas, entre ellas: promueve un mejor proceso de enseñanza y aprendizaje, entrega un input de calidad al estudiante y se adapta a las necesidades individuales, por lo mismo, los alumnos asumen más desafíos que en una comunicación cara a cara (Pennington, 1996).

Según Ferreira (2007), CALL se ha constituido en el área desde donde provienen los fundamentos teóricos y las proposiciones interdisciplinarias concretas sobre cómo integrar el computador en los procesos de enseñanza de lenguas. Además señala que esta interdisciplina en términos investigativos se interesa por averiguar sobre los diferentes usos y maneras de introducir la tecnología en el proceso de enseñanza-aprendizaje de lenguas y para ello se "nutre del trabajo empírico y teórico de otras áreas" (Ferreira, 2007: 273). Entre esas áreas se cuenta 
la Adquisición de Segundas Lenguas (ASL), Lingüística Aplicada e Informática educativa, entre otras. Sin embargo, no se trata de que esta interdisciplina sólo se nutra de otras áreas, sino que la investigación realizada en CALL lleva a resultados investigativos tangibles junto con nuevas perspectivas respecto del aprendizaje de una lengua y de la interacción entre el estudiante y la tecnología.

\subsubsection{Modalidades de enseñanza en el contexto de CALL}

De acuerdo a lo planteado en el punto anterior es necesario señalar que dentro del contexto de CALL existen diferentes modalidades de enseñanza de lenguas utilizando el computador. Ferreira (2007), menciona la modalidad e-learning y blended learning o b-learning, la primera se trata de aquella formación que se entrega de manera no presencial (on-line), y la segunda de aquella formación o enseñanza mixta, semipresencial, es decir, incluye tanto formación presencial como no presencial. En el siguiente apartado se describe con mayor detalle la modalidad semipresencial o blended learning debido a la relevancia que tiene para esta investigación.

\subsubsection{Modalidad semipresencial}

La modalidad e-learning cubre sólo una parte de las necesidades o requerimientos de los estudiantes, si a esto se suman los múltiples beneficios proporcionados por la tecnología en un contexto de enseñanza de lengua tradicional, entonces se logra una combinación entre estas dos modalidades (a distancia y presencial), combinación que se conoce con el nombre de blended learning o semipresencial.

Bartolomé (2004) señala que la historia del blended learning comienza con e-learning y principalmente con el fracaso (del e-learning), debido a que esta modalidad no respondió a las expectativas que había creado. Respecto al blended learning, señala que la definición más sencilla y precisa es aquella que describe un modo de aprender que combina la enseñanza presencial con la tecnología no presencial; este término también recibe el nombre de enseñanza semipresencial (Bartolomé, 2001, citado en Bartolomé, 2004).

Morales y Ferreira (2008), a partir de un estudio empírico en el que contrastaron dos modalidades de enseñanza (presencial/semipresencial) para el inglés como lengua extranjera, señalan que la modalidad semipresencial resultó más efectiva. Cuestión que también confirman García y Ferreira (2010) en un estudio basado en entrenamiento de estrategias de aprendizaje para el inglés como lengua extranjera en un contexto de aprendizaje combinado.

\subsubsection{La comunicación mediada por computador}

La comunicación mediada por computador (del inglés, Computer Mediated Com- 
munication, $\mathrm{CMC}$ ) puede definirse como cualquier forma de comunicación entre dos o más personas que interactúan mediante computadores a través de la red Internet. Profesores, investigadores y estudiantes se han interesado por la CMC, debido a que responde a necesidades comunicativas de quienes aprenden una lengua extranjera a través de la comunicación por Internet. En este sentido, Ferreira (2007) señala que la CMC se caracteriza tanto por una comunicación sincrónica como asíncrona, ambas altamente interactivas. Estas herramientas proporcionan un input activo entre quienes se comunican, por lo mismo, ya no es necesario siempre contar con un espacio físico (sala de clases) para practicar la lengua, sino de utilizar muchos de los entornos que hoy la CMC ofrece. Dentro de la CMC se pueden encontrar una serie de herramientas, tanto sincrónicas como asíncronas. A continuación se presentan algunas, las de mayor relevancia para este estudio.

\subsubsection{Chat sincrono}

Según lo que exponen Lamy y Hampel (2007) los programas de chat fueron las primeras herramientas de la CMC sincrónicas para el aprendizaje y enseñanza de lenguas. La ventaja de usar este recurso para enseñar una lengua es que los estudiantes lo utilizan en su vida cotidiana, por lo tanto no es una herramienta desconocida para ellos. Lamy y Hampel (2007) parafraseando a Weininger y Shield (2003) señalan que si bien el chat se asocia más con la escritura, su sincronía hace que el lenguaje utilizado se acerque más al discurso oral, por lo tanto el contexto que este medio proporciona es ideal para el aprendizaje de lenguas, ya que permite ensayar la interacción oral.

\subsubsection{Videocomunicación}

Jauregi, Canto y Gómez (2010) definen la videocomunicación como una herramienta que posibilita la comunicación oral sincrónica, ya que contiene en sí misma los elementos que antes faltaban en la comunicación mediada electrónicamente. Como es la percepción contextual, el contacto visual directo semejante al de cara a cara, información gestual, mecanismos de reparación dinámica en tiempo real, formas de evasión y, en general, la flexibilidad que normalmente se espera obtener entre los participantes en una conversación.

La Universidad de Utrecht (Holanda) a través del proyecto NIFLAR (Networked Interaction in Foreign Language Acquisition and Research, http://cms.hum. uu.nl/niflar/) tiene amplia experiencia en el uso de la videocomunicación para la enseñanza de ELE. Por ejemplo, estudios piloto entre estudiantes holandeses y españoles; y entre holandeses y chilenos, por mencionar sólo algunos, dan cuenta de lo útil que es contar con esta herramienta que facilita la comunicación virtual cara a cara entre los hablantes, proceso necesario para el proceso de adquisición/ 
aprendizaje de una lengua.

Las reacciones de los estudiantes según los estudios de Jauregi y Bañados (2008) y de Jauregi y Valdivia (2009) son positivas tanto en el uso de esta herramienta como también en el proceso de aprendizaje de ELE.

\subsubsection{Evaluación de la comunicación sincrónica}

Evaluar a estudiantes de segundas lenguas en una modalidad presencial es un procedimiento complejo, y aún más cuando se piensa medir los efectos de la comunicación sincrónica en los estudiantes que aprenden lenguas. Blake et al., (1999) realizaron una investigación respecto a la evaluación de la comunicación sincrónica a través de Internet como una posible técnica para enriquecer y mejorar la calidad del input comprensible. La meta de este experimento fue revisar si esta comunicación sincrónica tendría un efecto positivo en cuanto al desarrollo lingüístico de los estudiantes universitarios norteamericanos de español como segunda lengua. Al llegar a la etapa evaluativa de la comunicación sincrónica es cuando se presenta la dificultad sobre cómo evaluar los efectos de esta comunicación en cuanto al progreso lingüístico de los estudiantes de segundas lenguas. Evaluar la capacidad lingüística de los aprendientes es una actividad compleja, puesto que lo que producen en clases según Blake et al. "es una especie de interlenguaje lleno de errores y todo tipo de interferencias de la lengua materna" (1999: 13). Respecto a los instrumentos de evaluación para medir la competencia comunicativa, estos investigadores señalan que "faltan instrumentos de evaluación lingüística lo suficientemente adecuados y sutiles para captar la consolidación, no sólo gramatical sino también comunicativa que se va logrando paulatinamente" (1999: 13). En su estudio experimental, Blake et al. (1999) utilizaron dos tipos de tareas:

- Tareas abiertas

- Tareas cerradas

De estos dos tipos de tareas, resultó más efectiva la tarea cerrada, ya que los alumnos tuvieron que coincidir en una solución, aunque no única. Este tipo de tareas permite estimular la negociación entre las parejas de estudiantes (las tareas fueron cooperativas), debido a que los alumnos se fijan más en el código lingüístico y en el uso de estructuras lingüísticas que estén más allá de su capacidad, mientras que en las tareas abiertas no es necesario que los aprendientes negocien todas las confusiones comunicativas, puesto que el objetivo no es llegar a una solución.

El problema es cómo evaluar estas interacciones que sostienen los estudiantes a través de la red, ya que las medidas tradicionales de evaluación se centran en aspectos particulares de la gramática y, por lo mismo, no registran ningún efecto en este estudio de comunicación sincrónica con el programa de chat. Estas medidas 
no captan las sutilezas del desarrollo de los alumnos en vías del aprendizaje. Según Blake et al. (1999) los alumnos, a veces, mejoran, precisamente, cuando su actuación lingüística parece empeorar y es sólo después de un período de consolidación cuando el progreso lingüístico se empieza a notar en términos gramaticales, ya que el proceso de adquisición de una segunda lengua involucra varios factores, entre ellos, el dominio de la estructura lingüística, el conocimiento sociolingüístico, cultural etc., en resumidas cuentas se trata de una competencia comunicativa y no estrictamente gramatical.

El instrumento de evaluación más adecuado para el estudio realizado por Blake et al. (1999) fue un cuestionario sobre el experimento, el cual se les aplicó a los estudiantes. La particularidad de este instrumento reside en que los participantes pudieron comentar espontáneamente sus reacciones después de haber usado el programa de chat. Todos ellos señalaron que la actividad había sido de mucha utilidad y que la gran ventaja que presentaba era que podían comunicarse a través de la red. De manera que, cada uno tenía el tiempo suficiente para analizar lo dicho y escribir lo que quería decir, sin las presiones comunes a los métodos basados en la destreza oral, ya que estas presiones incomodan a los estudiantes de segundas lenguas, dejándolos en algunos momentos sin poder decir nada en el aula. Este instrumento fue más útil, de acuerdo a lo señalado por estos investigadores (Blake et al., 1999) que un examen de habilidades.

\subsection{Teorías de Adquisición de Segundas Lenguas}

Al momento de aprender una segunda lengua la mayoría de las personas lo hace en una sala de clases y no en un contexto de inmersión total, por lo que no están en contacto todo el tiempo con la lengua que aprenden. De este modo, la enseñanza de segundas lenguas ha dado lugar a la investigación en el campo de la Adquisición de Segundas Lenguas (ASL), en el que surgen dos paradigmas, el primero, basado en las teorías cognitivas provenientes de la psicología y la lingüística, y el segundo, influenciado por la teoría sociocultural.

\subsubsection{Modelo cognitivo de Adquisición de Segundas Lenguas (ASL)}

Este modelo se centra en los procesos cognitivos que involucran el aprendizaje y uso de la lengua, esto basado en los llamados modelos computacionales de aprendizaje de lenguas. Lamy y Hampel (2007) citando a Ellis (2000) señalan que la adquisición es el producto del procesamiento del input y del output, el input corresponde a las exposiciones del aprendiente a la lengua meta, mientras que el output corresponde a la producción que él o ella (aprendiente) realiza. De acuerdo a este planteamiento, no sólo el input es importante, sino también la interacción y el output. Como consecuencia de lo anterior se presenta la hipótesis de la inte- 
racción. Lamy y Hampel (2007) señalan que el lugar crucial para el desarrollo del lenguaje es la interacción entre los alumnos y otros hablantes, pero no sólo entre alumnos y hablantes más competentes en la lengua. Es en esta interacción donde se produce la negociación del significado para que los hablantes logren una comprensión mutua que dé lugar a una comunicación efectiva entre los interlocutores.

\subsubsection{Teoría sociocultural}

Desde finales de los años 90 ha habido un desarrollo general en ASL respecto a los aspectos sociales, esto influenciado por los planteamientos de Vygotsky (1978, citado en Warschauer, 2005) y luego de Leontiev (1981, citado en Lamy y Hampel, 2007). El aporte de la teoría sociocultural de Vygotsky ha sido de gran relevancia para la educación, ya que en sus desarrollos posteriores ha generado un impacto en los nuevos diseños curriculares y didácticos de la enseñanza. La enseñanza de lenguas no ha estado ajena a estos aportes, así también se plantean estas perspectivas socioculturales en el ámbito de CALL (Warschauer, 2005).

Uno de los aspectos más relevantes de la teoría sociocultural de Vygotsky es el concepto de mediación. Este término hace referencia a que la mente humana está mediada, como lo explica Warschauer "the notion that all human activity is mediated by tools or signs" (2005: 41). Esto implica que los diferentes recursos o herramientas, tales como: computadores, herramientas para escribir o el lenguaje mismo actúan como mediadores, en el sentido que no sólo facilitan la acción que podría ocurrir sin ellos, sino que son incluidos en los procesos de conducta o de comportamiento del ser humano. A través de este proceso de mediación, se altera el flujo y las funciones de las estructuras mentales, por lo tanto los seres humanos no actúan directamente en el mundo físico. Utilizan herramientas que le permiten regular sus relaciones con los otros y modificar las circunstancias o el mundo en que se desenvuelven.

El gran aporte de la teoría sociocultural de Vygotsky es el gran valor que le otorga al contexto social del que forma parte el individuo, ya que éste no se constituye aisladamente, sino en situaciones colectivas, el medio es el motor del aprendizaje. En este proceso de interacción se necesitan mediadores que guíen al niño para que desarrolle sus capacidades cognitivas.

\subsection{Enfoques de enseñanza de lenguas}

\subsubsection{Enfoque por tareas}

El enfoque por tareas constituye un movimiento de evolución, en palabras de Sheila Estaire (2007) "yo diría que natural, dentro del enfoque comunicativo de la enseñanza de lenguas extranjeras, ya que es lógico que un enfoque que comenzó 
en la década de los 70 evolucione y tome nuevas formas a medida que se continúa reflexionando sobre la lengua" (2007: 1). En este enfoque, la tarea es el punto de partida para la organización de la programación de una unidad didáctica (Estaire, 2007). Por lo tanto, se denomina tareas a "aquellas actividades para las cuales utilizamos la lengua en nuestra vida cotidiana” (Long, 1985, citado en Estaire, 2007).

A continuación se presenta el marco propuesto por Estaire $(2004,2007)$ para programar unidades didácticas a través de tareas.

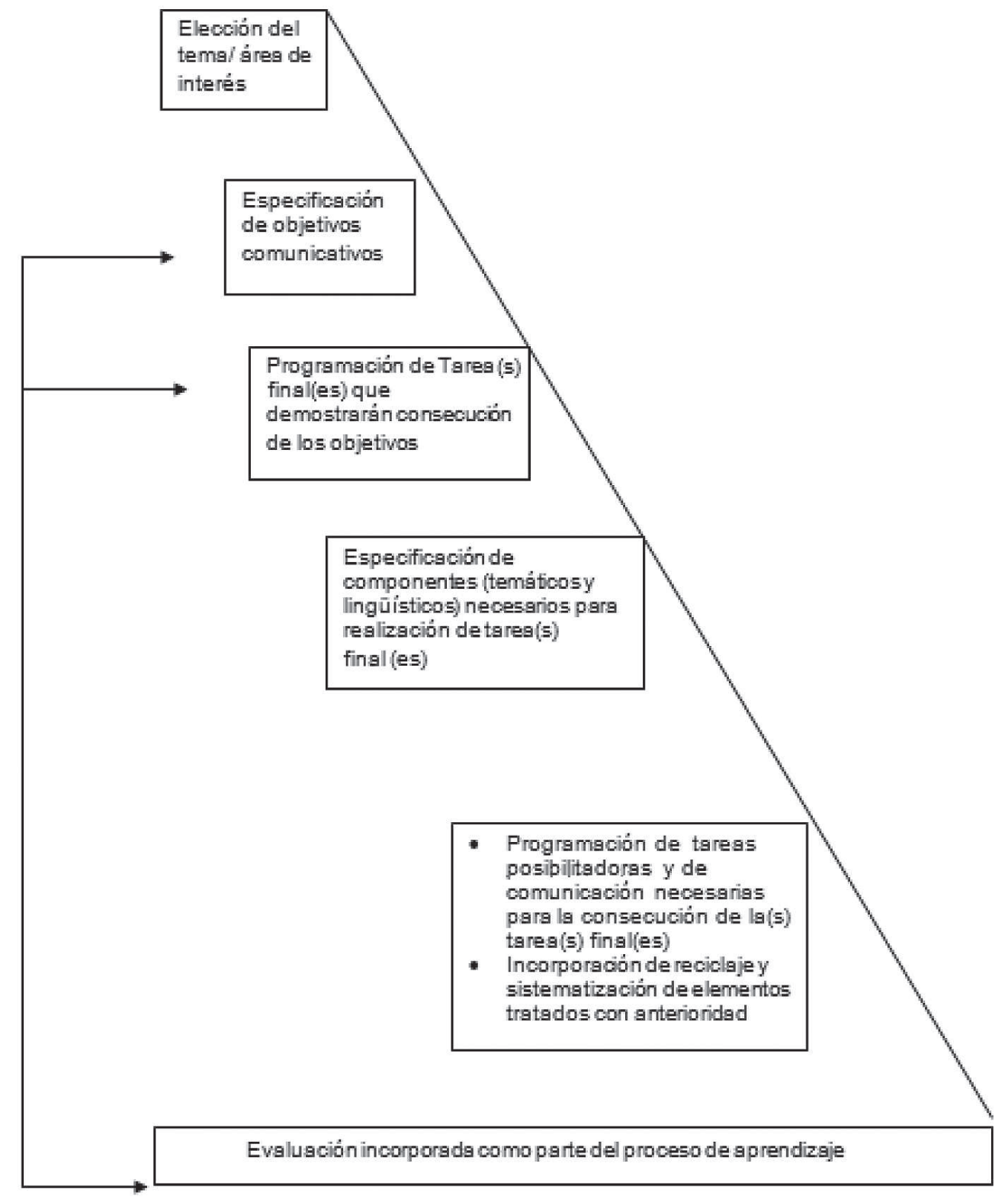

Figura 1. Marco de programación de una unidad didáctica (Estaire, 2004, 2007).

De los componentes presentados en este marco, en este estudio se utilizaron cuatro: elección del tema, especificación de objetivos comunicativos, componente 
lingüístico y tarea final.

A partir de la noción de tarea (Estaire, 2007), se aborda la tarea cooperativa intercultural. Jauregi, Canto y Gómez (2010) citan a Byram y Zárate (1998) para señalar lo que comprende el término intercultural. Ser intercultural es una actividad que consiste en ser capaz de interpretar y entender la perspectiva de los otros, pues aprender una lengua extranjera es más que poder transmitir un mensaje. Incluso más que interactuar exitosamente con hablantes nativos, es la habilidad de entender a otros en su especificidad nacional e histórica y llegar a entenderse uno mismo y su propia cultura. Se trata de que los estudiantes conozcan otras culturas y sean capaces de ver las diferencias entre la nueva y la suya propia. Por lo tanto, la tarea cooperativa intercultural se entiende como una actividad de aprendizaje que involucra un uso real (realidad simulada) de la lengua en cooperación y mutuo entendimiento (dimensión cultural) con el otro estudiante con quien se interactúa.

\subsubsection{Competencia comunicativa}

Jauregi y Sanz (en prensa) definen el término competencia comunicativa como el conocimiento y la destreza de reconocer e interpretar adecuadamente significados contextualizados y de hacer uso correcto, adecuado y coherente de elementos tanto lingüísticos como extralingüísticos en diferentes contextos comunicativos. Por lo tanto, no se trata sólo de que quienes aprenden una lengua la usen, sino que deben cumplir ciertas condiciones para que, efectivamente, se estén comunicando. La competencia comunicativa va más allá de los aspectos gramaticales y de léxico, involucra el comportamiento del hablante de acuerdo al contexto en que se desenvuelve. Canale y Swain (1980) definen la competencia comunicativa como la relación entre la competencia gramatical (conocimiento de las reglas gramaticales) y la competencia sociolingüística (reglas de uso de la lengua). Dentro de la competencia comunicativa se incluye también la competencia discursiva y estratégica como lo explica el modelo de Canale y Swain (1980).

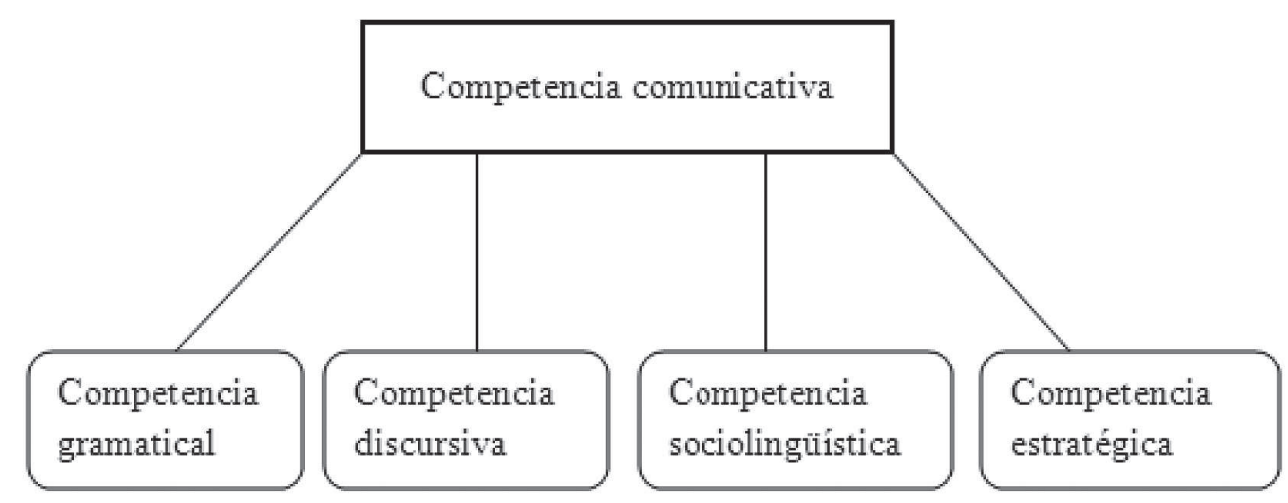

Figura 2. Modelo de competencia comunicativa (Canale y Swain, 1980). 
La competencia discursiva se refiere a la capacidad del hablante para producir e interpretar textos orales y escritos y la competencia estratégica a la habilidad para usar estrategias de comunicación, verbales y no verbales, que permitan superar dificultades que puedan surgir durante la comunicación.

\subsubsection{Aprendizaje cooperativo}

El aprendizaje cooperativo es un enfoque de enseñanza que aparece a mediados de los sesenta y que ha sido objeto de muchas investigaciones que muestran su efectividad en relación con el desarrollo afectivo, cognitivo y social del individuo, como lo señala Trujillo (2002) que existen datos que permiten afirmar que es el paradigma procesual, que agrupa al enfoque por tareas y cooperativo, el que de manera más eficiente crea la situación para que se satisfagan los requisitos cognitivos y situacionales para la adquisición de la lengua. El aprendizaje cooperativo se define como un "método de enseñanza que consiste en formar grupos pequeños y heterogéneos de aprendientes para trabajar juntos con el objetivo de una meta en común" (Kagan, 1994, citado en Urbano, 2004). Dentro de las características que son importantes de mencionar está el hecho de que se prioriza el trabajo grupal, siempre que se entienda que no se trata sólo de reunir a un grupo determinado de estudiantes de forma arbitraria, sino que debe haber un fin específico. Por ello, "el aprendizaje cooperativo se caracteriza por el tamaño y la composición del grupo, sus objetivos y 'roles', su funcionamiento, sus normas, y las destrezas sociales que lo crean, lo mantienen y lo mejoran" (Trujillo, 2002: 155). Este enfoque de enseñanza de lenguas ayuda en el desarrollo de destrezas sociales, resolución de problemas y perfecciona las destrezas comunicativas y lingüísticas (Trujillo, 2002). Los estudiantes asumen más responsabilidades respecto a su aprendizaje y al de sus compañeros. De este modo los propósitos generales de este enfoque se pueden resumir de la siguiente manera:

1) Fomentar la cooperación: Se aprende mejor colaborando -y no compitiendocon los compañeros.

2) Fomentar la integración de diferentes grupos entre sí, dentro y fuera de la clase.

3) Promover una enseñanza más reflexiva: basada en las habilidades y no tanto en la memorización de contenidos, para así mejorar el nivel de conocimientos de cada aprendiz, aumentando sus capacidades comunicativas y la interacción en clases.

Trujillo (2002) citando a Walters (2000) menciona cuatro modelos principales del aprendizaje cooperativo: 


\section{Jigsaw (Puzzle) \\ 2. Student Team Learning (Aprendizaje por Equipos de Estudiantes) \\ 3. Learning Together (Aprendiendo Juntos) \\ 4. Group Investigation (Investigación en Grupo)}

La diferencia que presenta cada uno de estos modelos se visualiza en la estructuración de la tarea, que es distinta en cada modelo. De acuerdo al objetivo planteado en este estudio, el interés se centra en una de estas técnicas, Aprendiendo Juntos (Learning Together) diseñada por Johnson y Johnson. Esta técnica se considera la más general de las técnicas de aprendizaje cooperativo, consiste en organizar grupos de dos a cinco integrantes que abordan una única tarea en la cual todos deben procurar el éxito del grupo y de cada individuo.

\section{ESTUDIO EXPERIMENTAL}

Con la finalidad de determinar si el uso de tareas cooperativas a través de la videocomunicación mejora la competencia comunicativa de estudiantes de ELE se llevó a cabo un estudio basado en el diseño experimental pretest/postest con grupo control. Para ello se delimitó la siguiente pregunta de investigación: ¿las tareas cooperativas interculturales desarrolladas a través de videocomunicación mejorarán la competencia comunicativa de los estudiantes de español como lengua extranjera? De acuerdo con esta pregunta se planteó la siguiente hipótesis:

Las tareas cooperativas interculturales desarrolladas a través de videocomunicación mejoran la competencia comunicativa de los estudiantes de español como lengua extranjera.

\subsection{Metodología}

\subsubsection{Muestra}

En este estudio participaron 16 estudiantes extranjeros, de los cuales el 62,5\% (10) eran hombres y el 37,5\% (6) mujeres, cuyas edades fluctuaban entre los 21 y los 45 años de edad. Estos sujetos eran de distintas nacionalidades, el $50 \%$ era de los Estados Unidos, 43,75 \% de Alemania y el 6,25\% de Haití. Todos ellos inscritos en el curso de Español para extranjeros que dicta el Departamento de Español de la Universidad de Concepción. Estos sujetos se distribuyeron aleatoriamente en los grupos, así a cada grupo le correspondió realizar tareas cooperativas interculturales en distintas modalidades, como se explica a continuación.

Grupo experimental: Los estudiantes de este grupo, después del pretest traba- 
jaron con el módulo de intervención lingüística que contemplaba la realización de cinco tareas cooperativas interculturales a través de videocomunicación (chat, cámara y voz) utilizando el programa Skype. Cada una de las tareas fue realizada en parejas.

Grupo control: Los estudiantes de este grupo trabajaron desarrollando las mismas tareas cooperativas interculturales que el grupo experimental, pero utilizando sólo el chat del programa Skype. Cada una de las tareas fue realizada en parejas.

\subsection{Procedimiento}

En relación con la hipótesis planteada, este estudio se desarrolló en diferentes etapas, las que se describen a continuación.

En primer lugar, con el objeto de averiguar el nivel de competencia de los estudiantes respecto al español, se aplicó un cuestionario. En segundo lugar, con la finalidad de evaluar el nivel de competencia lingüística que los estudiantes tenían de la lengua, se aplicó una prueba (en línea, (http://www.cfrd.cl/proyecto/espa$\mathrm{nol} / \mathrm{mod} /$ quiz/view.php?id=104) de proficiencia, instrumento elaborado por el Programa de Español como Lengua Extranjera de la Universidad de Concepción (ELE-UdeC). Esta prueba evalúa aspectos gramaticales, léxicos, producción escrita, comprensión auditiva y lectora; y está orientada hacia un nivel B1 de español. En tercer lugar, para medir el nivel de dominio de los estudiantes en el tópico de esta investigación, la competencia comunicativa, se aplicó un pretest que consistió en una entrevista que fue grabada, revisada y evaluada por dos profesoras. En esta entrevista se realizaron cinco preguntas sobre la temática del conflicto estudiantil chileno.

1. ¿Qué tal te ha parecido tu estadía en Chile?

2. ¿Qué has aprendido de la cultura chilena?

3. Uno de los temas que ha tenido a Chile en conflicto, relacionados con la educación, es el movimiento estudiantil ¿Qué piensas de este movimiento?

4. ¿Qué recomendarías para que el conflicto estudiantil se solucione?

5. Cuando regreses a tu país, ¿qué les contarás a tus amistades sobre el conflicto estudiantil chileno

En cuarto lugar se aplicó el proceso de intervención lingüística que consideró la realización de cinco tareas (una tarea por sesión) cooperativas interculturales 
en parejas de estudiantes. Para realizar estas tareas, el grupo experimental utilizó la videocomunicación y el grupo control el chat. Finalmente, una vez terminado el proceso de intervención se aplicó otra entrevista (postest) a los estudiantes, la cual también fue grabada, revisada y evaluada por dos profesoras de lenguas. Esta entrevista consistió en cinco preguntas sobre las temáticas de comidas típicas y lugares para visitar en Chile.

1. ¿Qué tal te ha parecido la cultura chilena?

2. ¿Qué has aprendido de la cultura gastronómica de Chile?

3. Uno de los aspectos que más atrae a los extranjeros a Chile son sus bellos paisajes ¿Qué piensas de los atractivos turísticos que Chile ofrece?

4. ¿Qué lugares de Chile recomendarías a otros extranjeros que visitan el país?

5. Cuando regreses a tu país, ¿qué les contarás a tus amistades sobre los lugares que conociste en Chile?

\subsection{Descripción de las entrevistas}

De acuerdo con la literatura especializada, un instrumento adecuado para evaluar competencia comunicativa es la entrevista. El procedimiento utilizado para llevar a cabo la entrevista se sustentó en los planteamientos de Bordón (2010) en cuanto al proceso de aplicación y revisión.

Para evaluar cada una de las entrevistas se confeccionó una escala de apreciación numérica de acuerdo a los criterios planteados por el Marco Común Europeo de Referencia para las Lenguas (MCER) para el nivel B1.

\begin{tabular}{|c|c|c|c|c|c|c|c|}
\hline Aspectos a evaluar: & \multicolumn{7}{|c|}{ Indicadores numéricos } \\
\hline \multicolumn{8}{|l|}{ Fluidez (20\%) } \\
\hline $\begin{array}{l}\text { - Se comunica de manera comprensible aun cuando realiza } \\
\text { pausas para continuar con la conversación }\end{array}$ & 1 & 2 & 3 & 4 & 5 & 6 & 7 \\
\hline $\begin{array}{l}\text { - Entiende lo dicho por un hablante nativo y se hace } \\
\text { entender con facilidad por el interlocutor. }\end{array}$ & 1 & 2 & 3 & 4 & 5 & 6 & 7 \\
\hline \multicolumn{8}{|l|}{ Interacción (20\%) } \\
\hline $\begin{array}{l}\text { - Participa activamente en la conversación, ajustándose a la } \\
\text { situación comunicativa. }\end{array}$ & 1 & 2 & 3 & 4 & 5 & 6 & 7 \\
\hline $\begin{array}{l}\text { - Es capaz de mantener o finalizar una conversación sobre un } \\
\text { tema de su interés. }\end{array}$ & 1 & 2 & 3 & 4 & 5 & 6 & 7 \\
\hline
\end{tabular}


Continuación Figura 3.

\begin{tabular}{|c|c|c|c|c|c|c|c|}
\hline \multicolumn{8}{|l|}{ Alcance $(20 \%)$} \\
\hline $\begin{array}{l}\text { - Tiene un repertorio lingüístico bastante amplio como para } \\
\text { desenvolverse y un vocabulario adecuado para expresarse } \\
\text { sobre temas de interés. }\end{array}$ & 1 & 2 & 3 & 4 & 5 & 6 & 7 \\
\hline \multicolumn{8}{|l|}{ Coherencia (20\%) } \\
\hline $\begin{array}{l}\text { - Es capaz de enlazar sus ideas para formar una secuencia } \\
\text { coherente. }\end{array}$ & 1 & 2 & 3 & 4 & 5 & 6 & 7 \\
\hline \multicolumn{8}{|l|}{ Precisión (20\%) } \\
\hline $\begin{array}{l}\text { - Utiliza correctamente (adecuados a la temática y a la } \\
\text { situación comunicativa) los elementos gramaticales y de } \\
\text { léxico. }\end{array}$ & 1 & 2 & 3 & 4 & 5 & 6 & 7 \\
\hline
\end{tabular}

Figura 3. Escala numérica para evaluar competencia comunicativa de acuerdo al MCER.

\subsection{Diseño del módulo de intervención lingüística}

En este proceso de intervención se elaboraron cinco tareas cooperativas interculturales, las que se diseñaron a partir de la propuesta de Estaire $(2004,2007)$ y del proyecto NIFLAR. Se consideraron tareas cerradas en los términos planteados por Blake et al. (1999), de modo que los estudiantes tuvieran que llegar a un resultado o conclusión después de cada proceso de interacción. A continuación se presenta el esquema utilizado en el diseño de cada una de las tareas.

\begin{tabular}{|l|l|}
\hline Elección del tema/ Título & \\
\hline Descripción de la tarea & \\
\hline Especificación de objetivos comunicativos e interculturales & \\
\hline Contenido lingüístico & \\
\hline Participantes & \\
\hline Especificaciones técnicas & \\
\hline Tarea final & \\
\hline
\end{tabular}

Figura 4. Esquema de elaboración de tareas (Estaire 2004, 2007; NIFLAR).

A las especificaciones descritas en la Figura 4 se agregó un ítem de instrucciones, donde se le entregó al estudiante cada uno de los pasos para que pudiera realizar las tareas.

La Figura 5 sintetiza cada una de las tareas y la modalidad en que fueron realizadas. De las cinco tareas, tres fueron realizadas en una modalidad no presencial y dos en modalidad presencial. El grupo experimental desarrolló estas tareas utilizando la videocomunicación y el grupo control el chat. Cada una de las temáticas 
planteadas en las tareas se determinó a partir de los intereses de los estudiantes.

\begin{tabular}{|l|l|}
\hline Sesiones & Modalidad \\
\hline Pretest (entrevista) & Presencial \\
\hline Tarea 1: Hidroaysén ¿̇un proyecto necesario? & Presencial \\
\hline Tarea 2: Conflicto estudiantil en Chile & No presencial \\
\hline Tarea 3: Comidas típicas chilenas & Presencial \\
\hline Tarea 4: Fiestas tradicionales & No presencial \\
\hline Tarea 5: Destinos para visitar & No presencial \\
\hline Postest (entrevista) & Presencial \\
\hline
\end{tabular}

Figura 5. Modelo blended learning.

\section{RESULTADOS}

Luego de aplicar los instrumentos a los grupos experimental y de control se procedió a analizar los datos obtenidos, tanto a través del pretest como del postest.

Cada una de las entrevistas se revisó de acuerdo a la escala de apreciación numérica (Figura 3) que presenta una serie de criterios con uno o más indicadores. Se consideraron cinco criterios: fluidez, interacción, alcance, coherencia y precisión, cada uno de ellos equivale a un $20 \%$ del valor total de esta entrevista que es de un $100 \%$.

De acuerdo a la hipótesis planteada, las Tablas I y II presentan los resultados que cada sujeto (S) del grupo control obtuvo en cada uno de los criterios. El criterio que presentó mayor avance en este grupo fue el de alcance con un 15,08 \%. La coherencia aumentó un $8,53 \%$, mientras que la fluidez e interacción aumentaron un 8,09 y $6,16 \%$ respectivamente, y la precisión aumentó un 8,09\%.

Tabla I. Resultados pretest grupo control.

\begin{tabular}{|l|l|l|l|l|l|l|}
\hline$S$ & $\begin{array}{l}\text { Fluidez } \\
\%\end{array}$ & $\begin{array}{l}\text { Interacción } \\
\%\end{array}$ & $\begin{array}{l}\text { Alcance } \\
\%\end{array}$ & $\begin{array}{l}\text { Coherencia } \\
\%\end{array}$ & $\begin{array}{l}\text { Precisión } \\
\%\end{array}$ & $\begin{array}{l}\text { Promedio } \\
\%\end{array}$ \\
\hline 1 & 81,50 & 77,10 & 70,00 & 77,10 & 70,00 & 75,14 \\
\hline 2 & 70,00 & 84,30 & 55,70 & 70,00 & 62,80 & 68,56 \\
\hline 3 & 77,10 & 84,30 & 70,00 & 70,00 & 70,00 & 74,28 \\
\hline 4 & 93,00 & 93,00 & 84,30 & 84,30 & 84,30 & 87,78 \\
\hline 5 & 77,10 & 84,30 & 70,00 & 84,30 & 62,80 & 75,70 \\
\hline 6 & 82,00 & 85,10 & 70,00 & 77,10 & 70,00 & 76,84 \\
\hline 7 & 77,10 & 100,00 & 84,30 & 84,30 & 84,30 & 86,00 \\
\hline 8 & 77,10 & 85,00 & 77,10 & 84,30 & 70,00 & 78,70 \\
\hline & 79,36 & 86,64 & 72,68 & 78,93 & 71,78 & 77,88 \\
\hline
\end{tabular}


Tabla II. Resultados postest grupo control.

\begin{tabular}{|l|l|l|l|l|l|l|}
\hline$S$ & $\begin{array}{l}\text { Fluidez } \\
\%\end{array}$ & $\begin{array}{l}\text { Interacción } \\
\%\end{array}$ & $\begin{array}{l}\text { Alcance } \\
\%\end{array}$ & $\begin{array}{l}\text { Coherencia } \\
\%\end{array}$ & $\begin{array}{l}\text { Precisión } \\
\%\end{array}$ & $\begin{array}{l}\text { Promedio } \\
\%\end{array}$ \\
\hline 1 & 85,10 & 100,00 & 92,10 & 92,10 & 85,00 & 90,86 \\
\hline 2 & 70,00 & 73,00 & 62,80 & 77,10 & 62,80 & 69,14 \\
\hline 3 & 81,00 & 85,10 & 77,10 & 77,10 & 77,10 & 79,48 \\
\hline 4 & 96,50 & 100,00 & 100,00 & 100,00 & 85,00 & 96,30 \\
\hline 5 & 81,00 & 84,30 & 70,00 & 77,10 & 70,00 & 76,48 \\
\hline 6 & 93,00 & 100,00 & 100,00 & 92,10 & 77,10 & 92,44 \\
\hline 7 & 93,00 & 100,00 & 100,00 & 92,10 & 84,30 & 93,88 \\
\hline 8 & 100,00 & 100,00 & 100,00 & 92,10 & 77,10 & 93,84 \\
\hline & 87,45 & $\mathbf{9 2 , 8 0}$ & $\mathbf{8 7 , 7 5}$ & $\mathbf{8 7 , 4 6}$ & 77,30 & 86,55 \\
\hline
\end{tabular}

Debido a que la muestra era pequeña se utilizó la prueba estadística $t$ de $s t u-$ dent. Esta prueba indica si la diferencia al comparar dos medias de un grupo, en dos momentos, es significativa. Los resultados obtenidos al contrastar el desempeño del grupo control en el pretest y postest arrojó un valor de $\mathrm{t}=3,8$ con un valor $\mathrm{p}=0,003$.

Las Tablas III y IV presentan los resultados obtenidos por el grupo experimental. Se puede señalar que el criterio que presentó un mayor avance fue el de precisión con un 17,14\%. El criterio de alcance tuvo un incremento de un 13,19\%, mientras que la fluidez y coherencia presentaron un avance de 10,78 y $10,44 \%$ respectivamente, y la interacción un 9,06\%.

Tabla III. Resultados pretest grupo experimental.

\begin{tabular}{|l|l|l|l|l|l|l|}
\hline$S$ & $\begin{array}{l}\text { Fluidez } \\
\%\end{array}$ & $\begin{array}{l}\text { Interacción } \\
\%\end{array}$ & $\begin{array}{l}\text { Alcance } \\
\%\end{array}$ & $\begin{array}{l}\text { Coherencia } \\
\%\end{array}$ & $\begin{array}{l}\text { Precisión } \\
\%\end{array}$ & $\begin{array}{l}\text { Promedio } \\
\%\end{array}$ \\
\hline 1 & 84,30 & 84,30 & 84,30 & 84,30 & 84,30 & 84,30 \\
\hline 2 & 84,30 & 81,00 & 70,00 & 77,10 & 70,00 & 76,48 \\
\hline 3 & 84,30 & 88,30 & 84,30 & 77,10 & 70,00 & 80,8 \\
\hline 4 & 100,00 & 93,00 & 92,20 & 92,10 & 70,00 & 89,46 \\
\hline 5 & 65,70 & 72,80 & 62,80 & 70,00 & 50,00 & 64,26 \\
\hline 6 & 77,10 & 84,30 & 70,00 & 84,30 & 62,80 & 75,7 \\
\hline 7 & 81,00 & 85,10 & 77,20 & 77,20 & 77,20 & 79,54 \\
\hline 8 & 84,30 & 84,30 & 84,30 & 77,20 & 70,00 & 80,02 \\
\hline & 82,63 & 84,14 & 78,14 & 79,91 & 69,29 & 78,82 \\
\hline
\end{tabular}


Tabla IV. Resultados postest grupo experimental.

\begin{tabular}{|l|l|l|l|l|l|l|}
\hline$S$ & $\begin{array}{l}\text { Fluidez } \\
\%\end{array}$ & $\begin{array}{l}\text { Interacción } \\
\%\end{array}$ & $\begin{array}{l}\text { Alcance } \\
\%\end{array}$ & $\begin{array}{l}\text { Coherencia } \\
\%\end{array}$ & $\begin{array}{l}\text { Precisión } \\
\%\end{array}$ & $\begin{array}{l}\text { Promedio } \\
\%\end{array}$ \\
\hline 1 & 100 & 100 & 100 & 100 & 92,1 & 98,42 \\
\hline 2 & 100 & 88,6 & 84,3 & 84,3 & 84,3 & 88,3 \\
\hline 3 & 93 & 100 & 100 & 100 & 84,3 & 95,46 \\
\hline 4 & 100 & 100 & 100 & 100 & 100 & 100 \\
\hline 5 & 77,1 & 72,8 & 70 & 70 & 70 & 71,98 \\
\hline 6 & 92,1 & 92,1 & 92,1 & 92,1 & 84,3 & 90,54 \\
\hline 7 & 92,1 & 100 & 92,1 & 92,1 & 92,1 & 93,68 \\
\hline 8 & 93 & 92,1 & 92,1 & 84,3 & 84,3 & 89,16 \\
\hline & 93,41 & 93,2 & 91,33 & $\mathbf{9 0 , 3 5}$ & $\mathbf{8 6 , 4 3}$ & $\mathbf{9 0 , 9 4}$ \\
\hline
\end{tabular}

El análisis estadístico muestra que en este grupo (experimental) hubo un avance más significativo que en el grupo control, así lo corrobora el valor entregado por el estadístico $\mathrm{t}=12,49$ con un $\mathrm{p}=0,000002$ (G. control $<\mathrm{G}$. experimental) al comparar los resultados obtenidos por ambos grupos.

El gráfico que se presenta a continuación muestra el avance general logrado por cada uno de los grupos en las dos mediciones (pretest y postest).

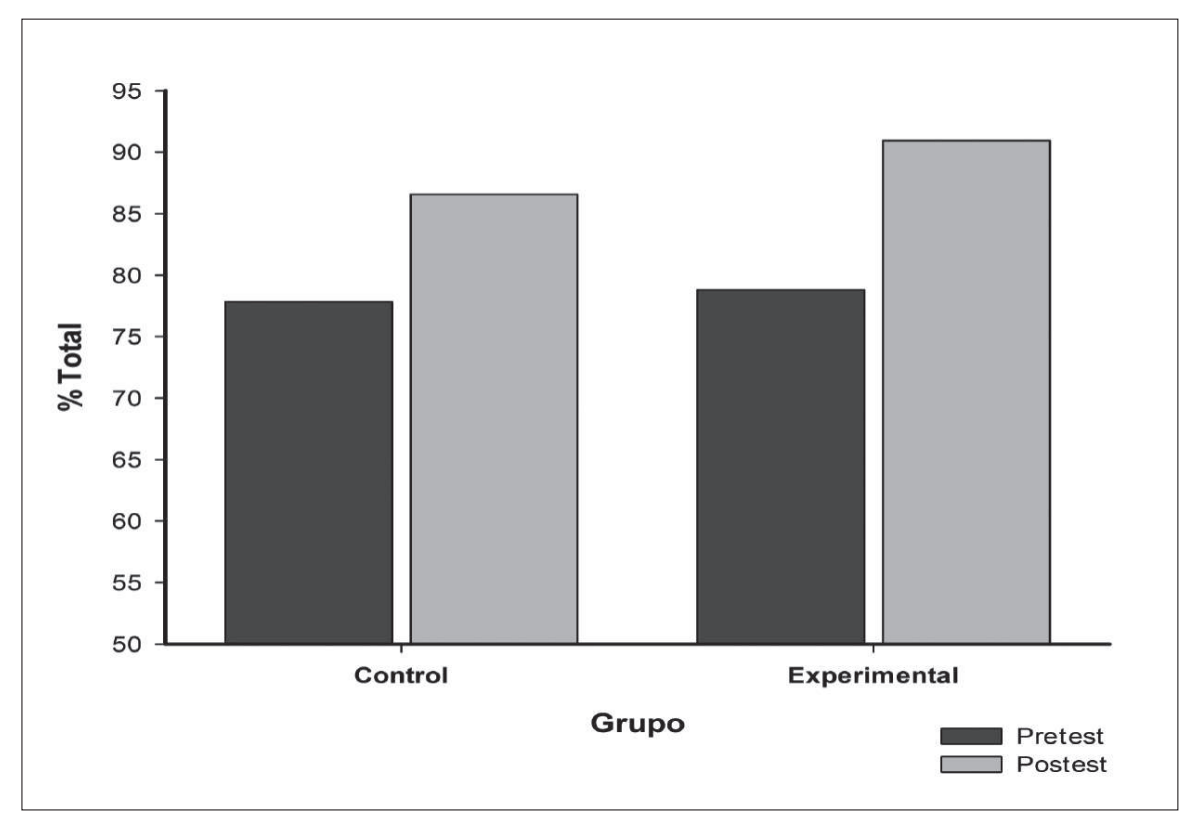

Gráfico 1. Avance logrado en pretest y postest.

Los resultados muestran que el mejoramiento de la competencia comunicativa 
para el grupo experimental fue de un $12,12 \%$, mientras que para el grupo control fue de un $8,67 \%$. Pese a que estas diferencias no son categóricas, permiten indicar cierta tendencia respecto a la efectividad de herramientas ofrecidas por la CMC, específicamente, la videocomunicación.

En consecuencia, hay que destacar el rol relevante que está teniendo la tecnología en el proceso de enseñanza-aprendizaje de lenguas. En este estudio, el uso de un recurso tecnológico como la videocomunicación fue un apoyo para mejorar la competencia comunicativa de estudiantes de ELE. Por lo tanto, resulta beneficioso incorporar herramientas de la CMC que ayuden en el desarrollo de habilidades específicas de la lengua.

\section{CONCLUSIONES}

La problemática sobre la competencia comunicativa ha sido poco investigada en la enseñanza de lenguas con tecnología por la dificultad que representa para los docentes llevarla a cabo, incluso en una modalidad presencial. El problema es aún más complejo cuando los estudiantes desarrollan gran parte de su trabajo utilizando el computador, porque el tema luego es ¿cómo medir el progreso que ha logrado el alumno? (Blake, 1999).

De acuerdo a lo planteado en el párrafo anterior, en este estudio se intentó medir el avance logrado por los estudiantes al trabajar en entornos que ofrece la $\mathrm{CMC}$, como el chat y la videocomunicación. Las investigaciones sobre uso de herramientas como videoconferencia, telecolaboración en la enseñanza del ELE sólo habían dado cuenta de las actitudes de los sujetos al usar estos recursos, pero no qué tan beneficioso resultaban en términos de aprendizaje el utilizar una u otra herramienta.

Los resultados obtenidos en este estudio muestran que fue más ventajoso realizar las tareas cooperativas interculturales en el entorno de videocomunicación que a través del chat, en cuanto al mejoramiento de la competencia comunicativa en ELE. Hay que señalar que el número de la muestra y el breve tiempo de aplicación de esta intervención (cinco sesiones) no permiten establecer conclusiones definitivas respecto al mejoramiento de la competencia comunicativa en ELE a través de la videocomunicación. Sin embargo, de acuerdo con los resultados, la videocomunicación parece el entorno ideal para fortalecer aspectos de precisión $(17,14 \%)$, que fue el que presentó un mayor avance durante este proceso de intervención. Esto puede deberse a que los estudiantes, al comunicarse en tiempo real (virtual) con su compañero, se preocupan de corregir tanto sus producciones orales como las de su interlocutor.

Es necesario señalar que hay pocos estudios en el ámbito de CALL que contrasten o comparen qué recursos ofrecidos por la tecnología son más o menos 
ventajosos para trabajar alguna habilidad lingüística determinada, sobre todo en este caso que se comparó el uso del chat versus el uso de videocomunicación. Por lo mismo, es necesario continuar investigando en esta línea, realizando intervenciones a mayor plazo para luego averiguar qué impacto tiene en el comportamiento comunicativo de quienes han aprendido o participado de un curso de lengua utilizando recursos como la videocomunicación.

\section{REFERENCIAS}

Bartolomé, Antonio. 2001. "Universidades en la Red. ¿¿Universidad presencial o virtual?” en Critica, LII (896), pp. 34-38.

Bartolomé, Antonio. 2004. "Blended learning. Conceptos básicos", en Píxel-Bit, Revista de Medios y Educación 23, pp. 7-20.

Bazo, Plácido. 1991. "Algunas técnicas para la evaluación comunicativa". ASELE ACTAS III [en línea]. Disponible en: http://cvc.cervantes.es/ensenanza/biblioteca_ele/asele/pdf/03/03_0415.pdf. Consulta: 28/06/2011.

Blake, Robert, Adam Karp y Eva Barela. 1999. "La evaluación de la comunicación sincrónica por Internet: El caso RTA, un programa de chat ", en REALE II, pp. 9-22.

Bordón, Teresa. 2010. "Evaluación de la lengua hablada", en Monográficos MarcoELE, No11, pp. 324-338.

Byram, M. y G. Zárate. 1998. Definitions, objectives and assessment of socio-cultural competence. Strasbourg. Council of Europe.

Canale, Michael y M. Swain. 1980. "Theoretical bases of communicative approaches to second language teaching and testing", en Applied Linguistics 1, pp. 1-47 [en línea]. Disponible en: https://segue.atlas.uiuc.edu/uploads/nppm/ CanaleSwain.80.pdf. Consulta: 27/06/2011.

Ellis, Rod. 2000. "Task-based Research and Language Pedagogy", en Language Teaching Research 4 (3), pp. 193-220.

Estaire, Sheila. 2004. "La programación de unidades didácticas a través de tareas", en Revista RedELE [en línea]. Disponible en: http://www.educacion.es/redele/ revista1/estaire.shtml. Consulta: 23/05/2011.

Estaire, Sheila. 2007. "La enseñanza de lenguas mediante tareas: principios y planificación de unidades didácticas" [en línea]. Disponible en: http://www.nebrija.es/espanolparainmigrantes/flash/ensenar/PDF/articulo-tareas.pdf. Consulta: 23/05/2011.

Ferreira, Anita. 2007. "Bases teóricas para un modelo B-learning de Enseñanza de Lenguas Extranjeras”. En J. Sánchez (Ed.): Nuevas Ideas en Informática Educativa, volumen 3. Santiago de Chile: LOM Ediciones, pp. 272-296.

García, Jaime y Anita Ferreira. 2010. "Entrenamiento en estrategias de aprendi- 
zaje de inglés como lengua extranjera en un contexto de aprendizaje combinado", en Revista Nebrija de Lingüistica Aplicada 8 (4), pp. 17-40.

Jauregi, Kristi y Emerita Bañados. 2008. "Virtual interaction through video-web communication: A step towards enriching and internationalizing language learning programs", en ReCALL 20 (2), pp. 183-207.

Jauregi, Kristi y David Sanz (en prensa). "Mis estudiantes son 'diginativos', ¿y los tuyos? el aprendizaje de la competencia comunicativa oral a través de entornos digitales: un reto posible", en Actas del XVII Congreso Internacional ASELE: Las destrezas orales en la enseñanza del español.

Jauregi, Kristi y Patricia Valdivia. 2009. "An initial approach to the study of intercultural communicative competence in interaction sessions conducted through video-web communication tools", en Presentation at the CMC SIG workshop of Eurocall: "Researching Computer Mediated Communication in Foreign Language Education: Issues and Methods". 23-25 abril. University of León, Spain.

Jauregi, Kristi, Silvia Canto y Carmen Ros. 2006. "La interculturalidad a través de la videoconferencia", en Actas del XVI Congreso Internacional ASELE: La competencia pragmática y la enseñanza del español como lengua extranjera. Oviedo, pp. 749-760.

Jauregi, Kristi, Silvia Canto y José Gómez. 2010. "Interacción virtual a través de la videocomunicación y mundos virtuales: dos estudios piloto", en MarcoELE Revista de Didáctica ELE 11, pp.1-17.

Juan, Olga. 2009. "Web 2.0, Comunicación y material didáctico digital para el aprendizaje del Español: El Aula Virtual de Español del Instituto Cervantes y su actualización", en RLA 47 (2), pp. 13-34.

Kagan, Spencer. 1994. Cooperative Learning. San Juan Capistrano, California: Kagan Cooperative Learning. Kagan publishing.

Lamy, Marie-Noëlle y Regine Hampel. 2007. Online Communication in Language Learning and Teaching. England: Palgrave Macmillan.

Leontiev, A. 1981. Psychology and the Language-learning Process, Oxford: Pergamon Press.

Long, Michael. 1985. A role for instruction in second language acquisition: Task based language training, en Hyltenstarm, K. y Pienemann, M. (eds.), Modelling and Assessing Second Language Acquisition. London, Multilingual Matters.

Ministerio de Educación, Cultura y Deporte. 2002. Marco común europeo de referencia para las lenguas: aprendizaje, enseñanza, evaluación. Madrid: Secretaria General Técnica del MECD y Grupo Anaya. Traducido y adaptado por el Instituto Cervantes.

Morales, Sandra y Anita Ferreira. 2008. "La efectividad de un modelo de aprendizaje combinado para la enseñanza del inglés como lengua extranjera: Estudio empírico", en RLA 46 (2), pp. 95-118. 
Pennington, Martha. 1996. The power of CALL. Houston: Athlestan Publications. Trujillo, Fernando. 2002. "Aprendizaje cooperativo para la enseñanza de la lengua". Revista Publicaciones de la Facultad de Educación y Humanidades Campus de Melilla 32, pp.147-162 [en línea]. Disponible en: http://meteco.ugr.es/lecturas/cooperacion.pdf. Consulta: 10/05/2011.

Urbano, Clara. 2004. "El aprendizaje cooperativo en discurso escrito en el aula de E/LE". Revista red ELE 1 [en línea]. Disponible en: http://www.educacion. gob.es/redele/revista1/urbano.shtml. Consulta: 08/06/2011.

Vygotsky, Leo. 1978. Mind in society: The development of higher psychological processes, Cambridge, MA: Harvard University Press.

Walters, Laurel. 2000. "Four Leading Models", en Harvard Education Letter's Research Online [en línea] Disponible en: http://www.edletter.org/past/ issues/2000-mj/models.shtml. Consulta: 10/05/2011.

Warschauer, Mark. 2005. "Sociocultural Perspectives on CALL", en J. Egbert y G. Mikel Petrie (eds.) CALL Research Perspectives. Mahwah, N.J.: Lawrence Earlbaum Associates Inc., pp. 41-51.

Weininger, Markus y Lesley Shield. 2003. "Promoting Oral Production in a Written Channel: An Investigation of Learner Language in MOO", en Computer Assisted Language Learning 16 (4), pp. 329-49. 